\title{
THE
}

\section{Cocatalyst Binding Effects in Organocatalytic Ring-Opening Polymerization of L-Lactide}

Oleg I. Kazakov

University of Rhode Island

Matthew K. Kiesewetter

University of Rhode Island, mkiesewetter@chm.uri.edu

Follow this and additional works at: https://digitalcommons.uri.edu/chm_facpubs

The University of Rhode Island Faculty have made this article openly available.

Please let us know how Open Access to this research benefits you.

This is a pre-publication author manuscript of the final, published article.

Terms of Use

This article is made available under the terms and conditions applicable towards Open Access

Policy Articles, as set forth in our Terms of Use.

\section{Citation/Publisher Attribution}

Kazakov, O. I., \& Kiesewetter, M. K. (2015). Cocatalyst Binding Effects in Organocatalytic Ring-Opening Polymerization of L-Lactide. Macromolecules, 48(17), 6121-6126. doi: 10.1021/acs.macromol.5b01140 Available at: http://dx.doi.org/10.1021/acs.macromol.5b01140 
Supporting Information for Cocatalyst Binding Effects in Organocatalytic Ring-Opening Polymerization of L-Lactide

Oleg I. Kazakov and Matthew K. Kiesewetter*

Department of Chemistry, University of Rhode Island, Kingston, RI 02881 USA

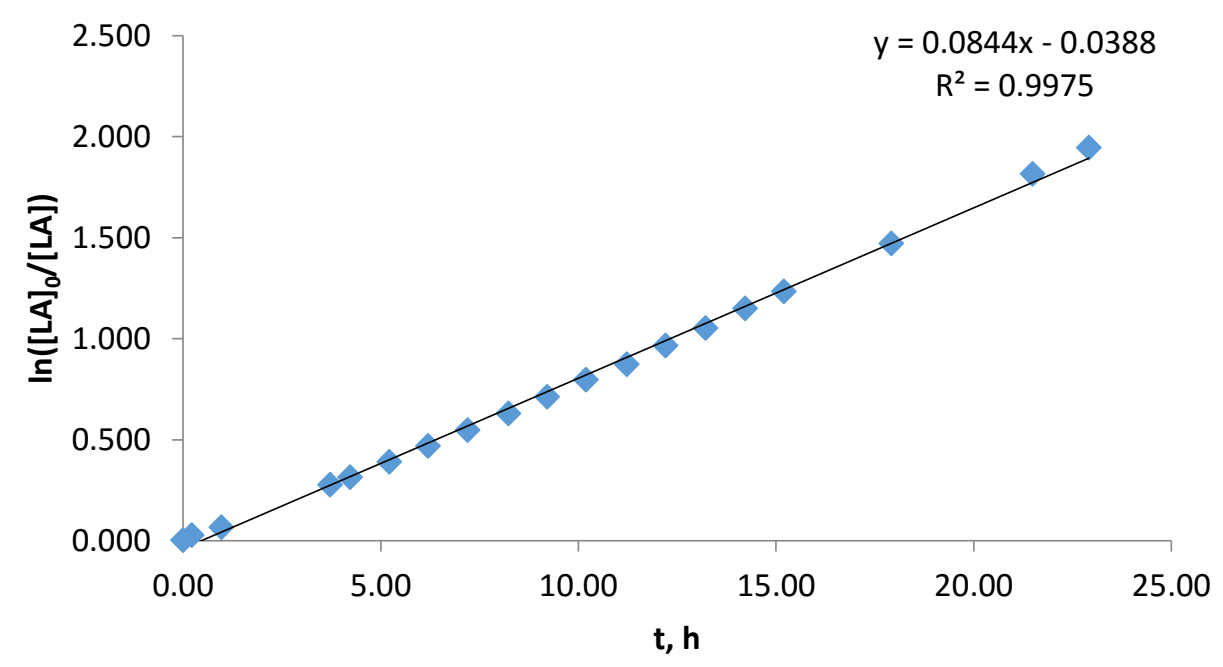

Figure S1. First order evolution of [LA] versus time in the 1/HMTETA catalyzed ROP of LA from benzyl alcohol in $\mathrm{CDCl}_{3}$. $[\mathrm{LA}]_{\mathrm{o}}=0.5 \mathrm{mmol} ;[\mathrm{HMTETA}]_{\mathrm{o}}=[1]_{\mathrm{o}}=0.025$ mmol; [benzyl alcohol $]_{\mathrm{o}}=0.010 \mathrm{mmol}$.

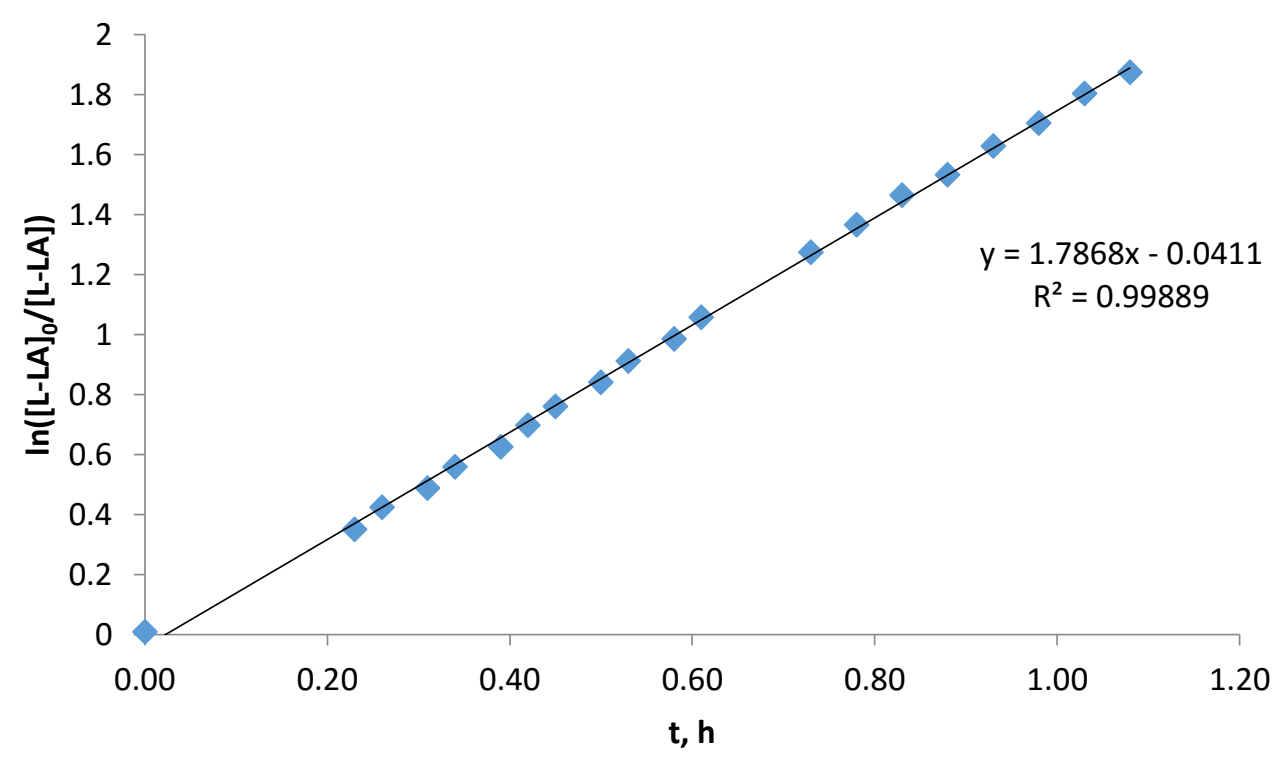

Figure S2. First order evolution of [LA] versus time in the $1 / \mathrm{Me}_{6} \mathrm{TREN}$ catalyzed ROP of LA from benzyl alcohol in $\mathrm{CDCl}_{3}$. $[\mathrm{LA}]_{\mathrm{o}}=0.5 \mathrm{mmol} ;\left[\mathrm{Me}_{6} \mathrm{TREN}\right]_{\mathrm{o}}=[1]_{\mathrm{o}}=0.050$ mmol; [benzyl alcohol $]_{\mathrm{o}}=0.010 \mathrm{mmol}$. 


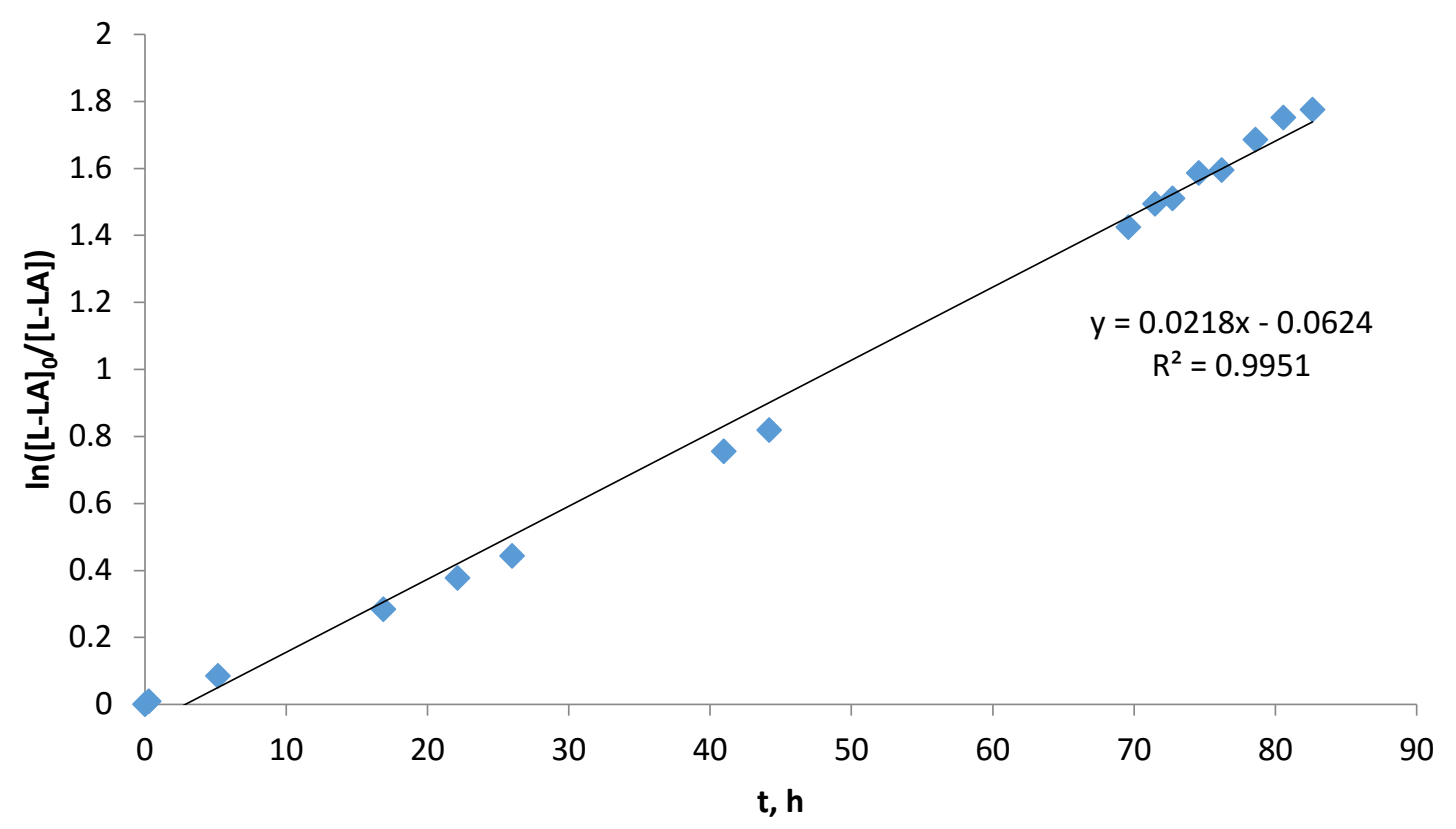

Figure S3. First order evolution of [LA] versus time in the 1/TMEDA catalyzed ROP of LA from benzyl alcohol in $\mathrm{CDCl}_{3}$. $[\mathrm{LA}]_{\mathrm{o}}=0.5 \mathrm{mmol} ;[\mathrm{TMEDA}]_{\mathrm{o}}=[1]_{\mathrm{o}}=0.050 \mathrm{mmol}$; $[\text { benzyl alcohol }]_{\mathrm{o}}=0.010 \mathrm{mmol}$.

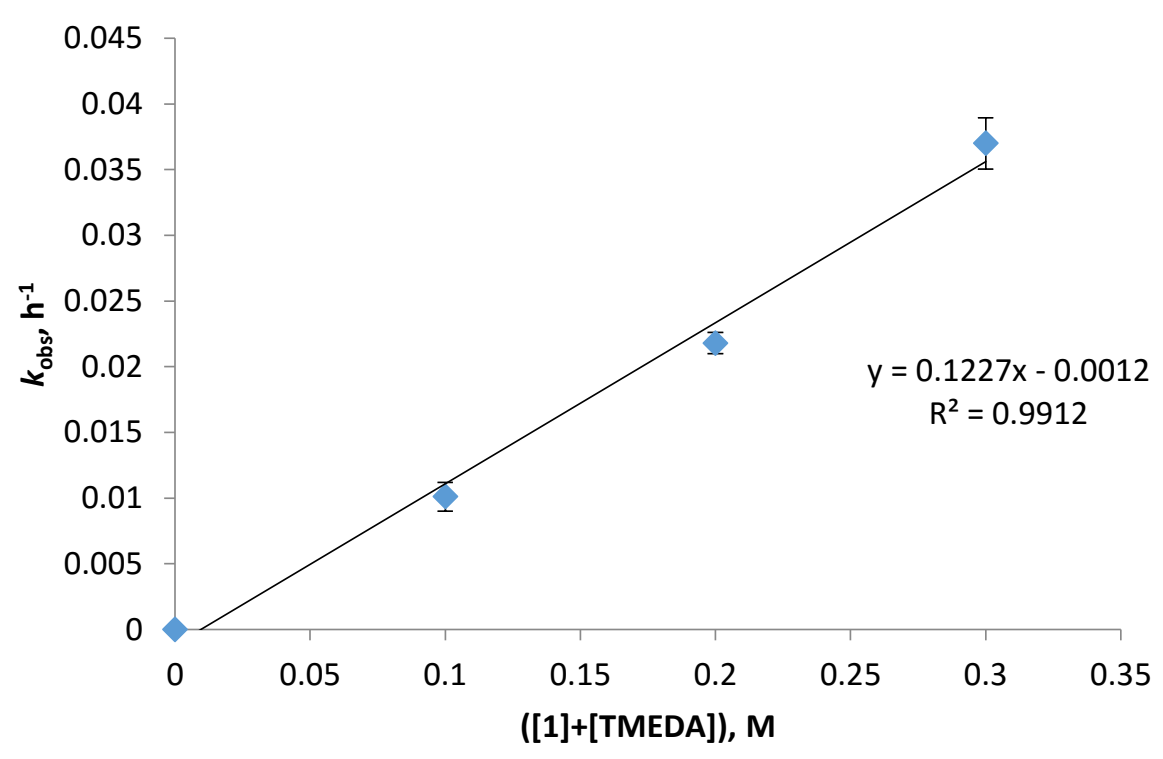

A) 


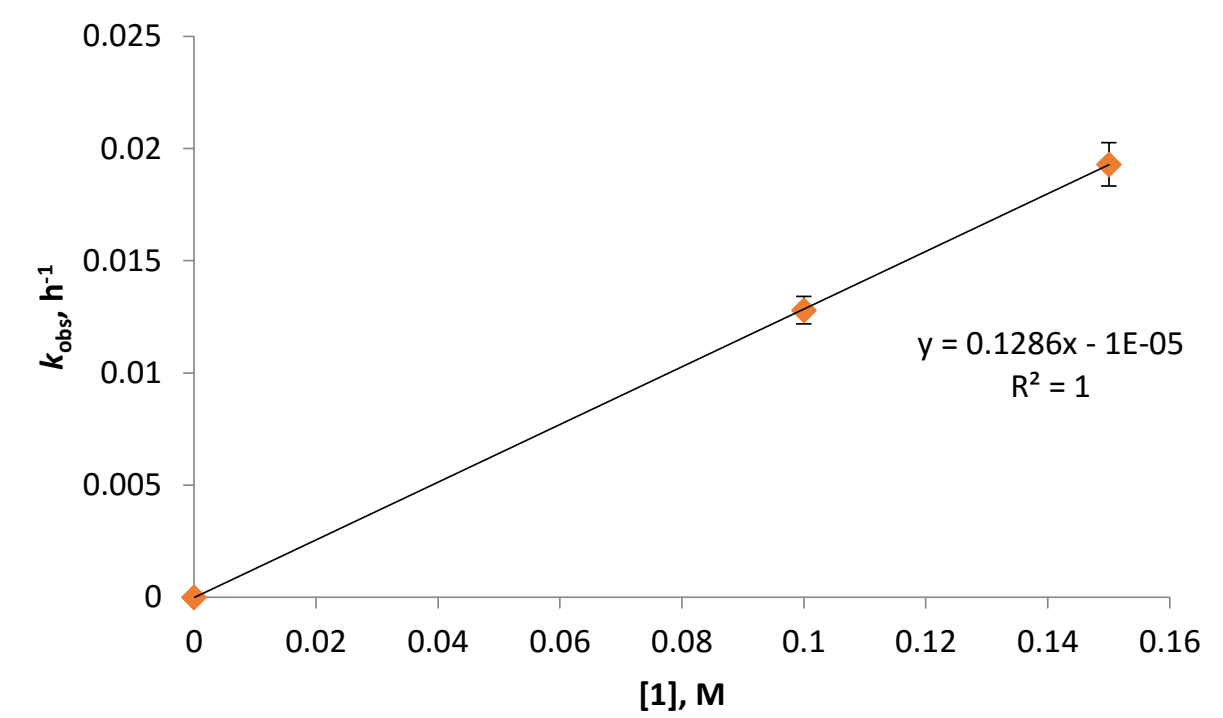

B)

C)

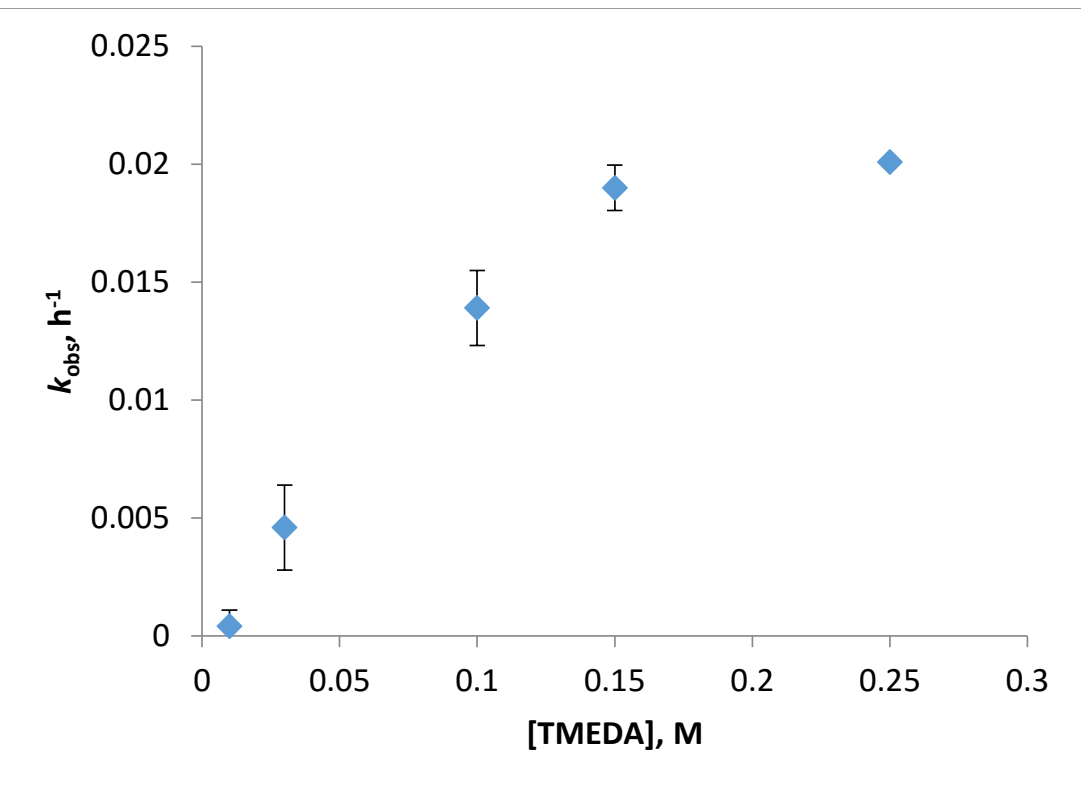

Figure S4. Observed rate constant $\left(\mathrm{k}_{\mathrm{obs}}\right)$ vs concentration plots for the 1/TMEDA catalyzed ROP of L-LA: A) $[\mathbf{1}]=[$ TMEDA], B) $[\mathbf{1}]>$ [TMEDA], [TMEDA] = $0.05 \mathrm{M}$, C) $\mathrm{k}_{\mathrm{obs}} \mathrm{vs}$ [TMEDA], [1] $=0.05 \mathrm{M}$ (bottom). 

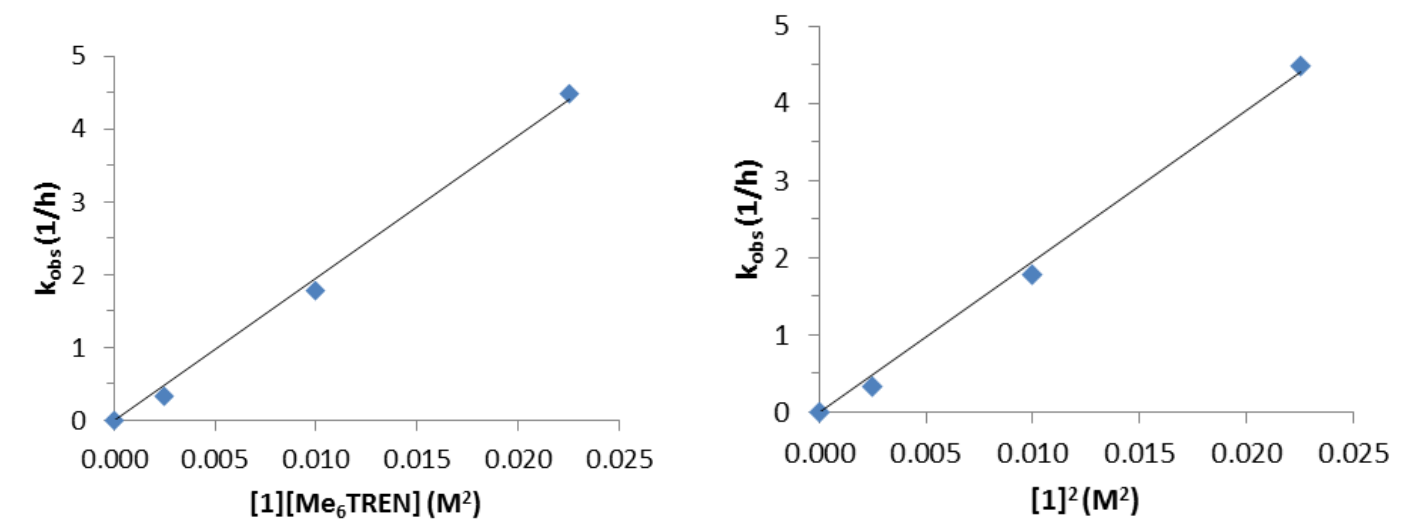

Figure S5. (left) Observed rate constant ( $\left.\mathrm{k}_{\mathrm{obs}}\right)$ vs [1][Me $6 \mathrm{MREN}_{6}$, and (right) observed rate constant $\left(\mathrm{k}_{\mathrm{obs}}\right)$ vs $[\mathbf{1}]^{2}$ for the $\mathbf{1} / \mathrm{Me}_{6} \mathrm{TREN}$ catalyzed ROP of L-LA.

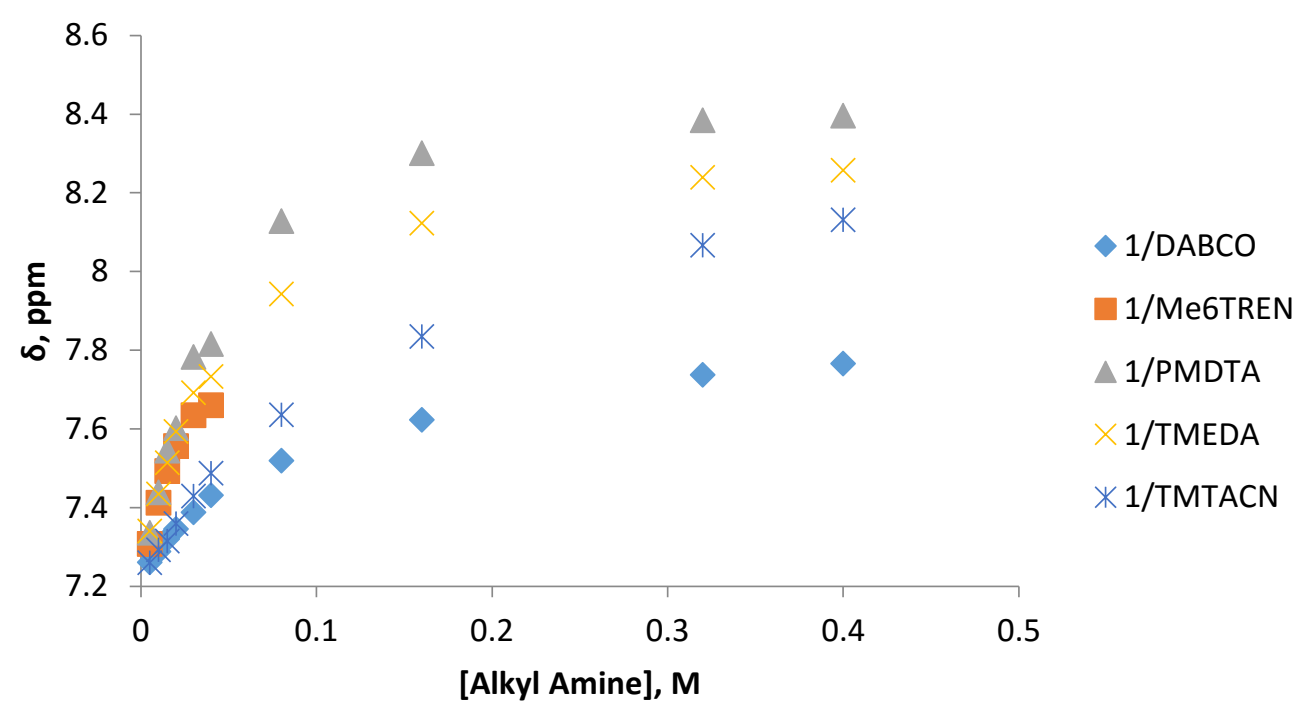

Figure S6. Binding curves for the association of 1 with alkylamine cocatalysts. The curve fitting method was used to extract binding constants, see Experimental Section. The chemical shift is of the ortho-protons of $\mathbf{1}$.

\section{Equation used for binding studies. ${ }^{1-3}$}

$\delta_{\mathrm{obs}}=\delta_{\mathrm{H}}-\left(\Delta \delta / 2[\mathrm{H}]_{\mathrm{o}}\right)\left\{[\mathrm{H}]_{\mathrm{o}}+[\mathrm{G}]_{\mathrm{o}}+1 / \mathrm{K}-\left(\left([\mathrm{H}]_{\mathrm{o}}+[\mathrm{G}]_{\mathrm{o}}+1 / \mathrm{K}\right)^{2}-4[\mathrm{H}]_{\mathrm{o}}[\mathrm{G}]_{\mathrm{o}}\right)^{1 / 2}\right\}$

Where: $\delta_{\text {obs }}$ is the observed chemical shift of the TU in the presence of base $\delta_{H}$ is the chemical shift of free TU in the absence of base $\Delta \delta$ is the difference in the chemical shift of host and complex, $\left(\Delta \delta=\delta_{\mathrm{C}}-\delta_{\mathrm{H}}\right)$ $\mathrm{K}$ is the binding constant, $\mathrm{K}_{\mathrm{eq}}$

The binding constants were determined by fitting the binding curve with the quadratic form of the binding equation shown above ( $\mathrm{K}_{\mathrm{eq}}$ and $\Delta \delta$ variables). ${ }^{1-2}$ The value of the binding constants determined from curve fitting are within experimental error of those 
determined from the slope of the linear (Lineweaver-Burke) forms of the binding equation. $^{4-5}$

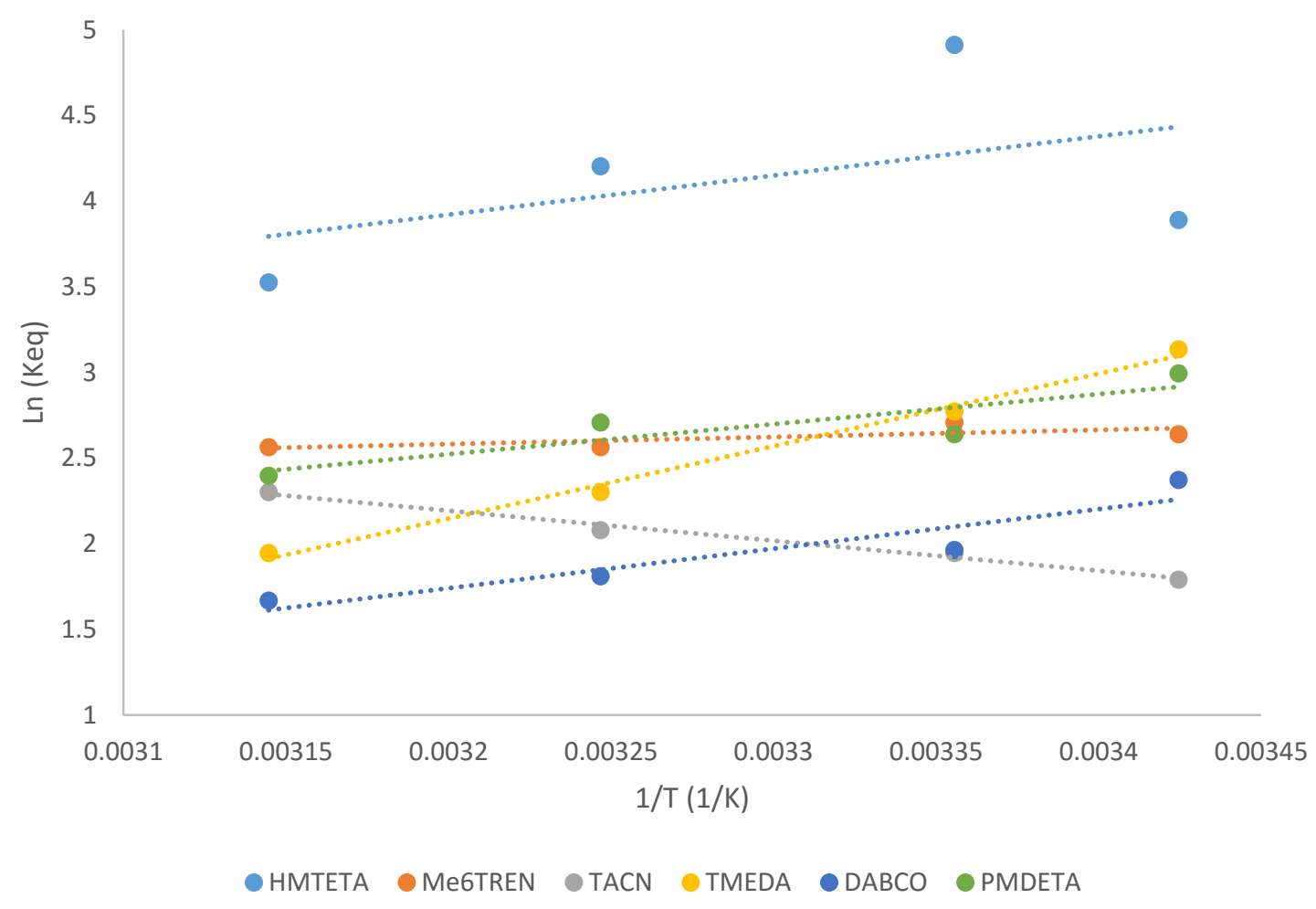

Figure S7. Temperature dependent binding.

\section{Computational Images of Cocatalyst Complexes.}

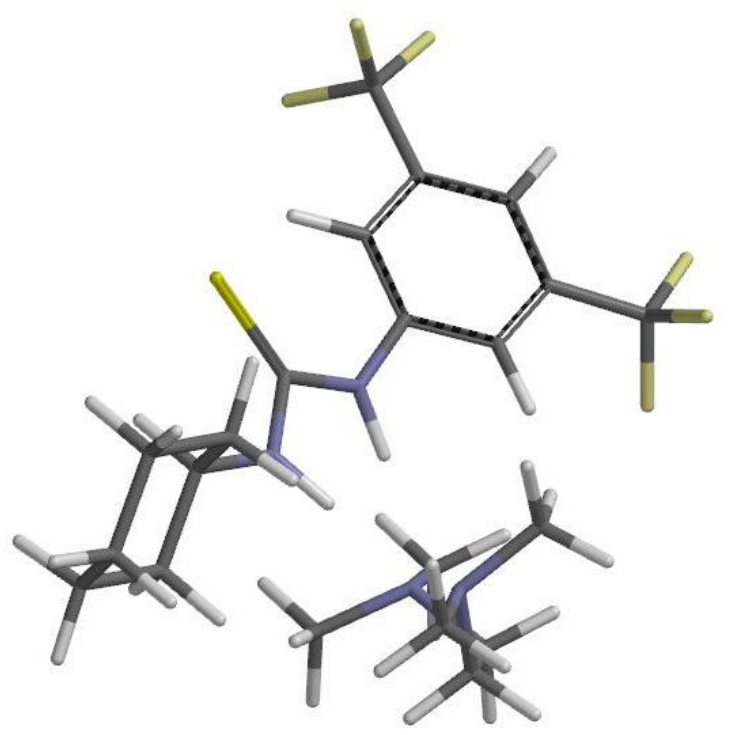

Figure S7. 1 + TMEDA. See Experimental Section for computational details and below for optimized coordinates. 


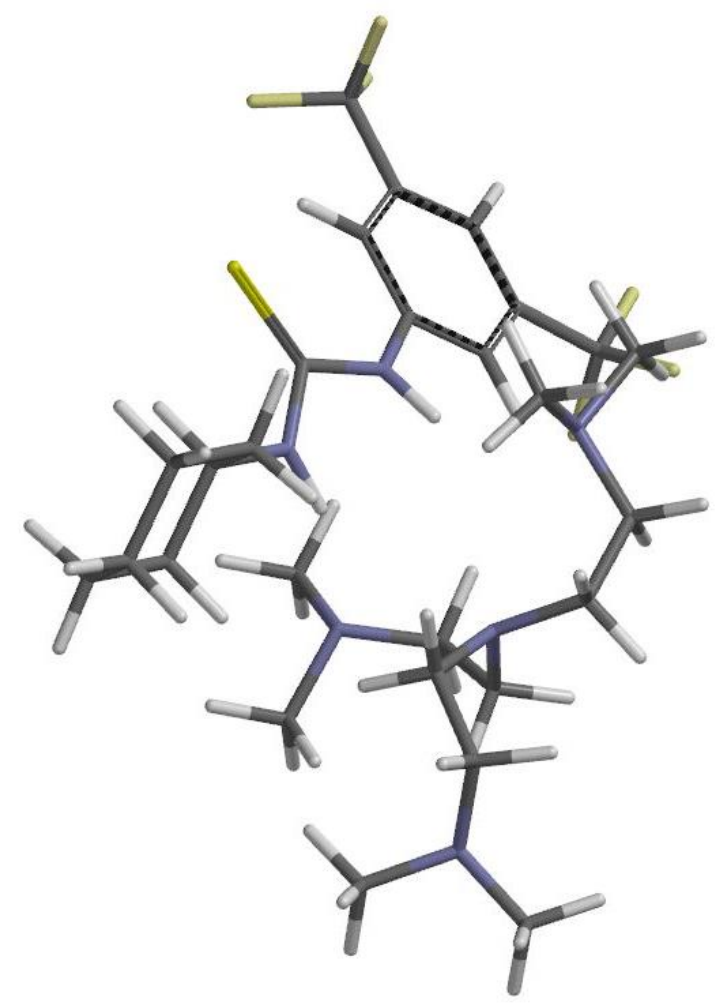

Figure S8. $1+\mathrm{Me}_{6}$ TREN. See Experimental Section for computational details and below for optimized coordinates.

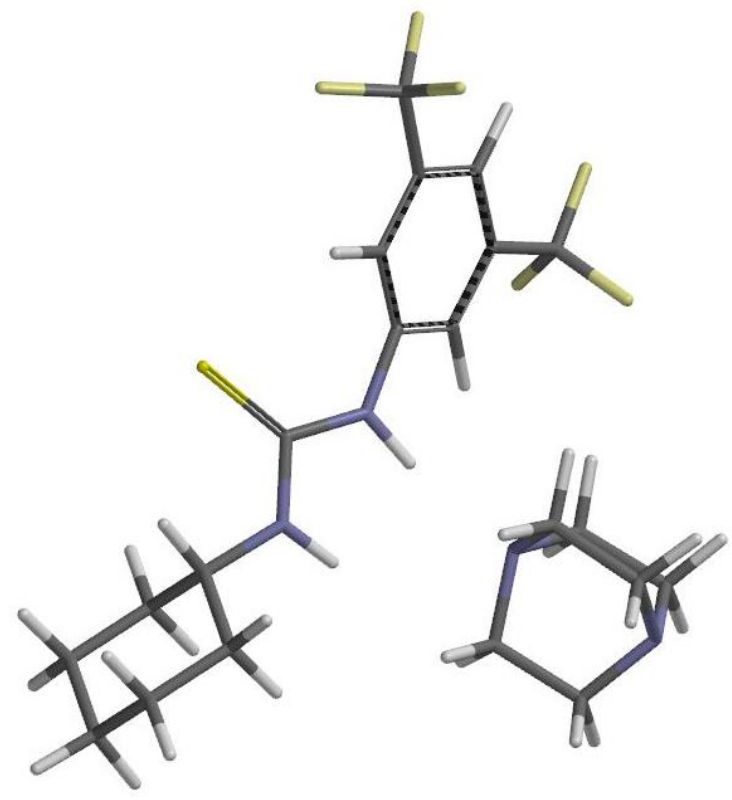

Figure S8. 1 + DABCO. See Experimental Section for computational details and below for optimized coordinates. 


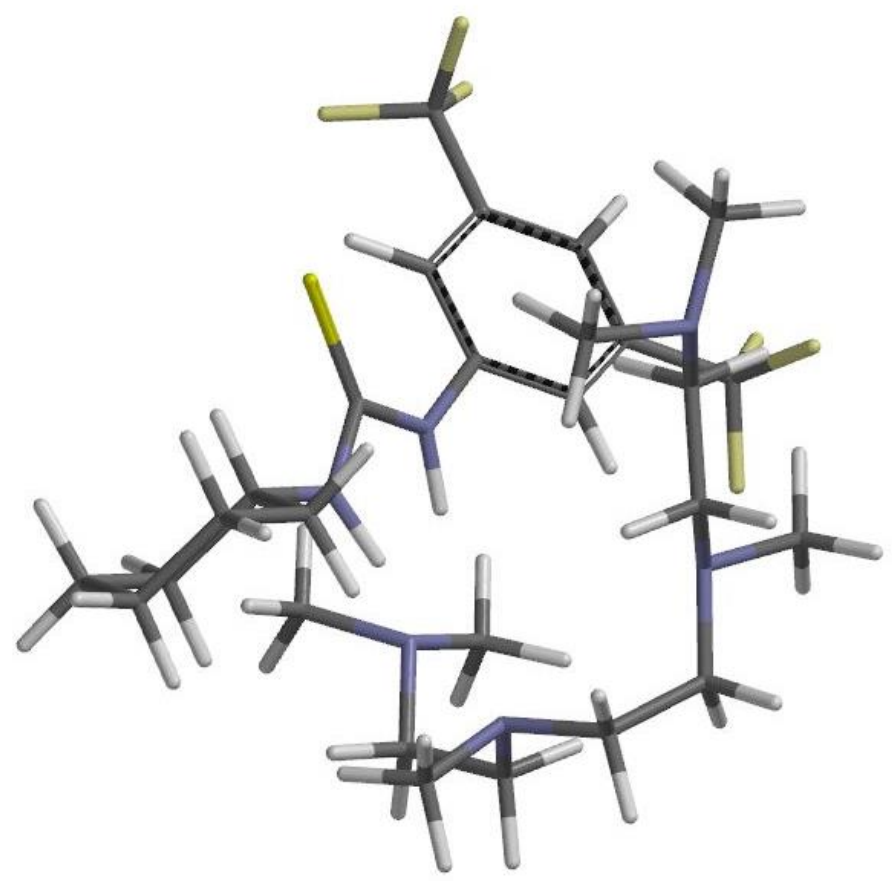

Figure S9. 1 + HMTETA. See Experimental Section for computational details and below for optimized coordinates.

Optimized Energies and Atomic Coordinates:

\section{$\underline{1+\text { HMTETA }}$}

\begin{tabular}{lcccc}
\multicolumn{5}{c}{ Cartesian Coordinates (Angstroms) } \\
Atom & X & Y & Z & \\
------------- \\
1 C C16 & 0.9894109 & -1.4727979 & -0.8215657 \\
2 S S1 & 0.6430497 & -3.1250735 & -0.7542201 \\
3 N N2 & 2.2567399 & -0.9719702 & -0.8520693 \\
4 H H10 & 2.3673275 & 0.0235565 & -0.6697293 \\
5 N N3 & 0.0487468 & -0.4735353 & -0.8389453 \\
6 H H27 & 0.3951613 & 0.4802393 & -0.9934503 \\
7 C C17 & -1.3536643 & -0.5391003 & -0.7540792 \\
8 C C18 & -4.1633420 & -0.3832505 & -0.6479231 \\
9 C C19 & -1.9865385 & 0.5674783 & -0.1699250 \\
10 C C20 & -2.1363603 & -1.5750872 & -1.2837411 \\
11 C C21 & -3.5270611 & -1.4876128 & -1.2149658 \\
12 C C22 & -3.3773418 & 0.6461909 & -0.1317388 \\
13 H H28 & -1.3716619 & 1.3511466 & 0.2606122 \\
14 H H30 & -1.6591248 & -2.4294477 & -1.7419244 \\
15 H H33 & -5.2436517 & -0.3196712 & -0.6245901 \\
16 C C23 & 3.4701700 & -1.7767561 & -0.9681170 \\
17 C C24 & 5.5695445 & -2.1977026 & -2.3280808 \\
18 C C25 & 5.5868573 & -2.5902270 & 0.1753813 \\
19 C C26 & 6.4142148 & -2.1636308 & -1.0458813 \\
20 C C27 & 4.3109634 & -1.7460253 & 0.3199323 \\
21 C C28 & 4.2993162 & -1.3453127 & -2.1899404 \\
22 H H36 & 5.2810725 & -3.2358982 & -2.5429145 \\
23 H H37 & 5.3070792 & -3.6475100 & 0.0713954 \\
24 H H38 & 6.7954007 & -1.1439239 & -0.8892658
\end{tabular}




\begin{tabular}{|c|c|c|c|}
\hline H H39 & 530 & 9347 & 26 \\
\hline $26 \mathrm{H} \mathrm{H} 40$ & 4.5825388 & -0.2874051 & -2.0865219 \\
\hline H H41 & 3.1194264 & -2.8008227 & -1.1250681 \\
\hline H H42 & 6.1596642 & -1.8546386 & -3.1858855 \\
\hline $\mathrm{H} \mathrm{H} 43$ & 6.1865864 & -2.5166078 & 1.0900585 \\
\hline H $\mathrm{H} 44$ & 7.2930945 & -2.8102422 & -1.1530313 \\
\hline H H45 & 3.7014172 & -2.1056411 & 1.1552688 \\
\hline $2 \mathrm{H} \mathrm{H} 46$ & 3.6812193 & -1.4246690 & -3.0915252 \\
\hline C C29 & -4.0219879 & 1.8357875 & 0.5235128 \\
\hline C C30 & -4.3547243 & -2.6287743 & -1.7476187 \\
\hline F F1 & -5.5818693 & -2.2155149 & \\
\hline F F2 & 1 & -3.22 & -2.8 \\
\hline F F3 & 1.54 & -3.587 & -0.8127692 \\
\hline F F4 & .2949743 & 2.0148487 & 0.1 \\
\hline F F5 & 4.0544452 & 1.7135871 & 1.8754090 \\
\hline F F6 & -3.34 & 2.9820225 & 0.2594708 \\
\hline N N1 & 2.6 & 2.5 & -0.0 \\
\hline $\mathrm{C} \mathrm{C} 1$ & 2.65 & & \\
\hline 3 H H4 & 10 & 3.9 & \\
\hline H H5 & 3.19 & 2.22 & 96 \\
\hline $5 \mathrm{C} \mathrm{C13}$ & 958 & 3.48 & -0.9 \\
\hline $5 \mathrm{C} C 3$ & 4.07 & 2.31 & -0.3 \\
\hline $7 \mathrm{H} \mathrm{H} 3$ & 4.54 & 1.59 & 281 \\
\hline H H11 & 068 & 1.92 & 824 \\
\hline $\mathrm{C} \mathrm{C} 4$ & 1.74 & 2.94 & -2.3 \\
\hline H H12 & & & \\
\hline $51 \mathrm{H} \mathrm{H} 13$ & 74 & 3.8 & 225 \\
\hline $52 \mathrm{C} \mathrm{C} 5$ & 1.30 & 3.27 & 2.0 \\
\hline 3 H H14 & 258 & 4.0 & 889 \\
\hline $4 \mathrm{H} \mathrm{H} 15$ & 0309 & 3.69 & 968 \\
\hline $5 \mathrm{~N} \mathrm{~N} 4$ & 0.5 & 618 & -2.4 \\
\hline $56 \mathrm{~N} \mathrm{~N} 5$ & 0.3 & & 2.17 \\
\hline $7 \mathrm{C} \mathrm{C7}$ & 0.56 & 1.28 & -3.6206611 \\
\hline 3 H H17 & 400 & & 922 \\
\hline $59 \mathrm{H} \mathrm{H} 21$ & & & \\
\hline $60 \mathrm{H} \mathrm{H} 22$ & 0.59 & 1.8 & 578 \\
\hline $61 \mathrm{C} \mathrm{C} 8$ & -0.6814797 & 2.9130532 & 3385 \\
\hline $2 \mathrm{H} \mathrm{H} 18$ & 3249 & 7529 & -3.2220017 \\
\hline H H23 & 024 & 85 & 5546 \\
\hline $64 \mathrm{H} \mathrm{H} 24$ & -1.5575433 & 2.2633073 & -2.4307914 \\
\hline 65 C C11 & & & 3.0334914 \\
\hline $6 \mathrm{H} \mathrm{H} 32$ & & & \\
\hline H H34 & & & \\
\hline 68 C C12 & -0.9 & 2.7 & 2.7 \\
\hline $9 \mathrm{H} \mathrm{H} 1$ & -1.2482672 & 3.56 & 2.0830226 \\
\hline $70 \mathrm{H} \mathrm{H} 1$ & -0.7831571 & 13 & 449 \\
\hline H H47 & -1.6979603 & 1.9967027 & 2.7363261 \\
\hline $72 \mathrm{H} \mathrm{H} 48$ & 1.0878049 & 3.7985586 & -0.4684969 \\
\hline 73 Н H49 & 2.6541594 & 4.3908863 & -1.0099409 \\
\hline $74 \mathrm{H} \mathrm{H} 7$ & & 3.25 & -0.34 \\
\hline $75 \mathrm{C} C 2$ & -0.06 & -0.10 & 3.1 \\
\hline 76 H H6 & -0.2826709 & -0.5014797 & 2.1411538 \\
\hline $\mathrm{H} \mathrm{H8}$ & -1.0186322 & 0.2058965 & 3.5825641 \\
\hline 78 N N6 & 3164 & -1.1243020 & 4.0439486 \\
\hline 79 C C6 & 1.5968539 & -1.8693305 & 3.4473450 \\
\hline $80 \mathrm{H} \mathrm{H} 9$ & 2.4082860 & -1.1931472 & 3.1644554 \\
\hline $81 \mathrm{H} \mathrm{H} 16$ & 1.2996867 & -2.4454297 & 2.5515433 \\
\hline $82 \mathrm{H} \mathrm{H} 20$ & 1.9997711 & -2.5700019 & 4.1857611 \\
\hline 83 C C9 & -0.5421762 & -2.0403384 & 4.5146340 \\
\hline
\end{tabular}




\begin{tabular}{|c|c|c|c|}
\hline $\mathrm{H} 2$ & -0.1055393 & -2.7459537 & 419 \\
\hline $85 \mathrm{H} \mathrm{H} 25$ & -1.0128186 & -2.6285078 & 3.7041436 \\
\hline $86 \mathrm{H} \mathrm{H} 26$ & -1.3292775 & -1.4814082 & 5.0312808 \\
\hline \multicolumn{4}{|l|}{$1+$ TACN } \\
\hline \multicolumn{4}{|c|}{ Cartesian Coordinates (Angstroms) } \\
\hline Atom & $X$ & $\mathrm{Z}$ & \\
\hline $1 \mathrm{~N} \mathrm{~N} 1$ & -3.9631626 & -2.3050019 & -1.2539444 \\
\hline $2 \mathrm{C} \mathrm{C} 1$ & -4.8886767 & -2.7814741 & -2.2730449 \\
\hline $3 \mathrm{H} \mathrm{H} 3$ & -5.5106800 & -3.5872821 & -1.8699328 \\
\hline $4 \mathrm{H} \mathrm{H} 4$ & -4.3243359 & -3.1905683 & -3.1182340 \\
\hline $5 \mathrm{H} \mathrm{H} 5$ & -5.5550754 & -1.9903955 & -2.6683066 \\
\hline $6 \mathrm{C} \mathrm{C} 2$ & -4.6096232 & -1.8619147 & -0.0203063 \\
\hline 7 H H6 & -5.6039747 & -2.3225152 & 0.0330960 \\
\hline $8 \mathrm{H} \mathrm{H} 7$ & -4.7757403 & -0.7694037 & -0.0149363 \\
\hline $9 \mathrm{C} \mathrm{C} 3$ & -3.8746646 & -2.2736638 & 1.26 \\
\hline $10 \mathrm{H} \mathrm{H} 8$ & -3.8 & -3.3 & 2164 \\
\hline $11 \mathrm{H} \mathrm{H} 9$ & -4.5039855 & -1.9550676 & 2.1068505 \\
\hline $12 \mathrm{C} \mathrm{C} 4$ & -2.9845535 & -1.3540467 & -1.7997892 \\
\hline $13 \mathrm{H} \mathrm{H} 10$ & -2.7762733 & -0.5859165 & -1.0547378 \\
\hline $14 \mathrm{H}$ H11 & -3.4073089 & -0.8360211 & -2.6780426 \\
\hline 15 C C5 & -1.6507800 & -1.9842827 & -2.2135349 \\
\hline $16 \mathrm{H} \mathrm{H} 12$ & -1.1336123 & -1.2736685 & -2.8697925 \\
\hline $17 \mathrm{H} \mathrm{H} 13$ & -1.8 & -2.8 & 7716 \\
\hline $18 \mathrm{~N} \mathrm{~N} 2$ & -2.5 & -1.7 & 3723 \\
\hline $19 \mathrm{~N} \mathrm{~N} 3$ & -0.7 & -2.271 & -1.0 \\
\hline $20 \mathrm{C} \mathrm{C} 6$ & -1.458 & -2.7161697 & 1.2812582 \\
\hline $21 \mathrm{H} \mathrm{H} 16$ & -1.6359694 & -3.5752216 & 1.9584852 \\
\hline $22 \mathrm{H} \mathrm{H} 17$ & -0.5164651 & -2.2576495 & 1.6011561 \\
\hline $23 \mathrm{C} \mathrm{C} 7$ & -1.2717620 & -3.2679231 & -0.1336680 \\
\hline $24 \mathrm{H} \mathrm{H} 18$ & 350 & -4.1095125 & -0.0553601 \\
\hline $25 \mathrm{H} \mathrm{H} 19$ & -2 & -3 & -0 . \\
\hline $26 \mathrm{C} \mathrm{C} 9$ & -2.38 & -1.10 & 2.78 \\
\hline $27 \mathrm{H} \mathrm{H} 1$ & -2.4718091 & -1.8446681 & 3.6018546 \\
\hline $28 \mathrm{H} \mathrm{H} 20$ & -3.1523584 & -0.3409928 & 2.9316040 \\
\hline $29 \mathrm{H} \mathrm{H} 21$ & -1.4072375 & -0.6139913 & 2.8755968 \\
\hline $30 \mathrm{C} \mathrm{C} 10$ & -0.3709184 & 1.6522465 & 0.3890683 \\
\hline 31 N N4 & -1.7127224 & 1.4628076 & 0.5120385 \\
\hline $32 \mathrm{H} \mathrm{H} 24$ & -2 & 743 & 0.5790606 \\
\hline $33 \mathrm{~N} \mathrm{N5}$ & 0.31 & 0.47 & 0.1 \\
\hline $34 \mathrm{H} \quad \mathrm{H} 23$ & -0.2 & 1215 & -0.05 \\
\hline $35 \mathrm{~S} \mathrm{S1}$ & 0.3784710 & 3.1575981 & 0.5371844 \\
\hline $36 \mathrm{C} \mathrm{C} 11$ & 1.7128331 & 0.2948509 & 0.1134650 \\
\hline $37 \mathrm{C} \mathrm{C} 12$ & 4.4596054 & -0.3015849 & -0.0435930 \\
\hline $38 \mathrm{C} \mathrm{C} 13$ & 2.5710317 & 1.1149817 & -0.6330972 \\
\hline $39 \mathrm{C} \mathrm{C} 14$ & 2.2432870 & -0.8095529 & 0.7840138 \\
\hline $40 \mathrm{C} \mathrm{C} 15$ & 244 & -1.1 & 0.6858338 \\
\hline $41 \mathrm{C} \mathrm{C} 16$ & 3.9322063 & 0.8202717 & -0.6889279 \\
\hline $42 \mathrm{H} \mathrm{H} 22$ & 2.1729769 & 1.9686009 & -1.1632310 \\
\hline $43 \mathrm{H} \mathrm{H} 26$ & 1.5910922 & -1.4331372 & 1.3862344 \\
\hline $44 \mathrm{H} \mathrm{H} 29$ & 5.5147685 & -0.5351550 & -0.1154477 \\
\hline 45 C C17 & -2.7057116 & 2.5371246 & 0.5439967 \\
\hline $46 \mathrm{C} \mathrm{C} 18$ & -4.4297349 & 3.8392205 & -0.7904108 \\
\hline 47 C C19 & -4.7568961 & 3.4715049 & 1.6987798 \\
\hline $48 \mathrm{C}$ C20 & -5.4518274 & 3.6477860 & 0.3404729 \\
\hline 49 C C 21 & -3.7303318 & 2.3278134 & 1.6697620 \\
\hline
\end{tabular}




$\begin{array}{lccc}\text { 50 C C22 } & -3.4024262 & 2.6982701 & -0.8204078 \\ \text { 51 H H25 } & -3.9029409 & 4.7934909 & -0.6477641 \\ \text { 52 H H30 } & -4.2443530 & 4.4054508 & 1.9697636 \\ \text { 53 H H31 } & -6.0635677 & 2.7572660 & 0.1300492 \\ \text { 54 H H32 } & -4.2580361 & 1.3728265 & 1.5166967 \\ \text { 55 H H33 } & -3.9058246 & 1.7560227 & -1.0849440 \\ \text { 56 H H34 } & -2.1403753 & 3.4499352 & 0.7527893 \\ \text { 57 H H35 } & -4.9412813 & 3.9077456 & -1.7585613 \\ \text { 58 H H36 } & -5.4983013 & 3.2842202 & 2.4854917 \\ \text { 59 H H37 } & -6.1436403 & 4.4987462 & 0.3773559 \\ \text { 60 H H38 } & -3.2086290 & 2.2558407 & 2.6319109 \\ \text { 61 H H39 } & -2.6431580 & 2.8818684 & -1.5896707 \\ \text { 62 C C23 } & 4.8568765 & 1.7480689 & -1.4331844 \\ \text { 63 C C24 } & 4.0915529 & -2.3847066 & 1.3275451 \\ \text { 64 F F1 } & 5.3363983 & 2.7196332 & -0.6264127 \\ \text { 65 F F2 } & 5.9269385 & 1.0900910 & -1.9361994 \\ \text { 66 F F3 } & 4.2354730 & 2.3572002 & -2.4653152 \\ \text { 67 F F4 } & 3.6783539 & -3.4756434 & 0.6285434 \\ \text { 68 F F5 } & 3.6082901 & -2.5308782 & 2.5819912 \\ \text { 69 F F6 } & 5.4354727 & -2.4444453 & 1.3964676 \\ \text { 70 C C8 } & 0.5642315 & -2.6952970 & -1.6307742 \\ \text { 71 H H2 } & 0.9890776 & -1.8953792 & -2.2459015 \\ \text { 72 H H14 } & 0.4902535 & -3.6060394 & -2.2524422 \\ \text { 73 H H15 } & 1.2628477 & -2.8976087 & -0.8149270\end{array}$

\section{$\underline{1+\mathrm{Me}_{6} \text { TREN }}$}

\begin{tabular}{|c|c|c|c|}
\hline \multirow[b]{2}{*}{ Atom } & \multicolumn{3}{|c|}{ Cartesian Coordinates (Angstroms) } \\
\hline & $\mathrm{X}$ & $\mathrm{Z}$ & \\
\hline $1 \mathrm{C} \mathrm{C} 16$ & -0.3565532 & -1.9654632 & 0.2387363 \\
\hline $2 \mathrm{~S}$ S1 & -1.1207598 & -3.4475029 & 0.4941631 \\
\hline $3 \mathrm{~N} \mathrm{~N} 2$ & 0.9719516 & -1.8466738 & -0.0174063 \\
\hline $4 \mathrm{H} \mathrm{H} 10$ & 1.3074932 & -0.9566372 & -0.3934779 \\
\hline $5 \mathrm{~N} \mathrm{~N} 3$ & -0.9757238 & -0.7252759 & 0.3006581 \\
\hline $6 \mathrm{H} \mathrm{H} 27$ & -0.3722192 & 0.0499826 & 0.5924125 \\
\hline 7 C C17 & -2.3084752 & -0.3440481 & 0.0937992 \\
\hline $8 \mathrm{C} \mathrm{C} 18$ & -4.9143951 & 0.6752252 & -0.3453424 \\
\hline $9 \mathrm{C} \mathrm{C} 19$ & -2.5400110 & 1.0365476 & -0.0393070 \\
\hline $10 \mathrm{C} \mathrm{C} 20$ & -3.4058028 & -1.2145768 & -0.0150334 \\
\hline $11 \mathrm{C} \mathrm{C} 21$ & -4.6815944 & -0.6950182 & -0.2357005 \\
\hline $12 \mathrm{C} \mathrm{C} 22$ & -3.8227716 & 1.5347176 & -0.2418054 \\
\hline $13 \mathrm{H} \mathrm{H} 28$ & -1.7038945 & 1.7235529 & 0.0202816 \\
\hline $14 \mathrm{H} \mathrm{H} 30$ & -3.2512939 & -2.2800549 & 0.0797111 \\
\hline $15 \mathrm{H} \mathrm{H} 33$ & -5.9121122 & 1.0599851 & -0.5086353 \\
\hline $16 \mathrm{C} \mathrm{C} 23$ & 1.9047760 & -2.9753131 & -0.0214730 \\
\hline 17 C C24 & 3.9118105 & -4.0150346 & -1.1615347 \\
\hline $18 \mathrm{C} \mathrm{C} 25$ & 3.5684324 & -4.3331403 & 1.3300990 \\
\hline 19 C C26 & 4.5990173 & -4.1959931 & 0.1998898 \\
\hline $20 \mathrm{C} \mathrm{C} 27$ & 2.5911907 & -3.1492103 & 1.3452993 \\
\hline $21 \mathrm{C} \mathrm{C} 28$ & 2.9328753 & -2.8305365 & -1.1526160 \\
\hline $22 \mathrm{H} \mathrm{H} 36$ & 3.3649591 & -4.9328453 & -1.4169447 \\
\hline $23 \mathrm{H} \mathrm{H} 37$ & 3.0021816 & -5.2645983 & 1.1939824 \\
\hline $24 \mathrm{H}$ H38 & 5.2411063 & -3.3255996 & 0.3987345 \\
\hline 25 H H39 & 3.1309476 & -2.2249158 & 1.5961858 \\
\hline $26 \mathrm{H} \mathrm{H} 40$ & 3.4964247 & -1.8965405 & -1.0226784 \\
\hline $27 \mathrm{H} \quad \mathrm{H} 41$ & 1.2995863 & -3.8676532 & -0.2122285 \\
\hline
\end{tabular}




\begin{tabular}{|c|c|c|c|}
\hline $28 \mathrm{H} \mathrm{H} 42$ & 4.6595808 & -3.8707964 & -1.9502402 \\
\hline $29 \mathrm{H} \mathrm{H} 43$ & 4.0723748 & -4.4156428 & 2.3001553 \\
\hline H H44 & 5.2591200 & -5.0710342 & 0.1762892 \\
\hline H H45 & 1.8230726 & -3.2920537 & 2.1125161 \\
\hline H H46 & 2.4117507 & -2.7557165 & -2.1139955 \\
\hline C C29 & -3.9911292 & 3.0223452 & -0.3838011 \\
\hline C C30 & -5.8441155 & -1.6536240 & -0.2865727 \\
\hline F F1 & 6.9221760 & -1.1022743 & -0.8889782 \\
\hline $36 \mathrm{~F} \mathrm{~F} 2$ & .5367901 & -2.7824334 & -0.9584769 \\
\hline $37 \mathrm{~F} \mathrm{F3}$ & -6.2325557 & -2.0272279 & 0.9540142 \\
\hline 38 F F4 & -5.2849372 & 3.3972487 & -0.3886396 \\
\hline $\mathrm{F} F 5$ & -3.3818722 & 3.6903011 & 0.63 \\
\hline F F6 & -3.4330444 & 3.4828813 & -1.5279053 \\
\hline N N1 & 2.67 & 1.5729156 & 0.5387792 \\
\hline $\mathrm{C} \mathrm{C} 1$ & 2.4560060 & 2.2769173 & 1.8008238 \\
\hline $\mathrm{H} \mathrm{H} 4$ & 2.9756586 & 3.2532645 & 1.8337622 \\
\hline 4 H H5 & 2.9036435 & 1.6771309 & 2.5998396 \\
\hline $5 \mathrm{C} \mathrm{C} 13$ & 7650 & 2.4043496 & -0.6619079 \\
\hline $5 \mathrm{C} \mathrm{C} 3$ & 3.90 & 0.77 & 0.5 \\
\hline $\mathrm{H} \mathrm{H} 3$ & 3.81 & 0.0 & 992 \\
\hline H H11 & 3.9299761 & 3593 & 1796 \\
\hline $\mathrm{C} \mathrm{C} 4$ & 1.3290279 & 1.9414201 & -1.5757468 \\
\hline H $\mathrm{H} 12$ & 1.1942213 & 2.7081642 & -2.3651848 \\
\hline $51 \mathrm{H} \mathrm{H} 13$ & 6557 & 1.9235519 & -0.9922123 \\
\hline $52 \mathrm{C} \mathrm{C} 5$ & 0.9979294 & 2.5652812 & 2.1696056 \\
\hline $53 \mathrm{H} \mathrm{H} 14$ & 4077 & & 452 \\
\hline $54 \mathrm{H} \mathrm{H} 15$ & 664 & 073 & 948 \\
\hline C C6 & 5.24 & 1.51 & 0.7 \\
\hline $56 \mathrm{H}$ H6 & 5.99 & 0.8 & 054 \\
\hline H H16 & 5.1170011 & 2.2891301 & 1.4946283 \\
\hline N N4 & $1.4 \mathrm{C}$ & 0.60 & -2.1 \\
\hline 59 N N5 & 0.1108668 & 211 & 2.2981623 \\
\hline 0 N N6 & 3458 & 2.1697795 & -0.4862453 \\
\hline $1 \mathrm{C} \mathrm{C7}$ & 2.62 & 0.55 & -3.0 \\
\hline H H17 & & -0 . & 588 \\
\hline H H21 & 265 & 2945 & 7624 \\
\hline $4 \mathrm{H} \quad \mathrm{H} 22$ & 2.4975802 & 1.2371232 & -3.9578844 \\
\hline C C8 & 0.2784606 & 0.2019085 & -2.8696812 \\
\hline 5 H H18 & 0.0455305 & 0.8600357 & -3.7268575 \\
\hline $7 \mathrm{H} \mathrm{H} 23$ & -0.5735378 & 0.2176121 & -2.1878272 \\
\hline $8 \mathrm{H} \quad \mathrm{H} 24$ & 0.3922826 & -0.8189360 & -3.2462111 \\
\hline 9 C C9 & 6.3856359 & 1.2148333 & -1.3900349 \\
\hline H H9 & 6.6 & 1.7 & -2 \\
\hline H H25 & 614 & 6208 & 526 \\
\hline H H26 & 7.2800528 & 0.7354826 & -0.9471517 \\
\hline C C10 & 6.6751609 & 3.2558425 & -0.1697362 \\
\hline $74 \mathrm{H} \mathrm{H} 20$ & 7.5785419 & 2.9141576 & 0.3701598 \\
\hline $75 \mathrm{H} \mathrm{H} 29$ & 6.1713257 & 4.0042144 & 0.4494834 \\
\hline $76 \mathrm{H} \mathrm{H} 31$ & 7.0001824 & 3.7443355 & -1.0935897 \\
\hline 7 C C11 & 0.6890679 & 0.3261788 & \\
\hline $8 \mathrm{H} \mathrm{H} 2$ & 9571 & -0.46 & 3.23 \\
\hline H H32 & 0.9924531 & 0.6695158 & 4.1201041 \\
\hline $\mathrm{H} \mathrm{H} 34$ & 1.5535040 & -0.1122512 & 2.6122861 \\
\hline C $\mathrm{C} 12$ & -1.1598265 & 1.8303244 & 2.8902341 \\
\hline $82 \mathrm{H} \mathrm{H} 1$ & -1.6168627 & 2.6163775 & 2.2837463 \\
\hline $83 \mathrm{H} \mathrm{H} 19$ & -1.0278637 & 2.2212039 & 3.9152612 \\
\hline $84 \mathrm{H} \mathrm{H} 47$ & -1.8559628 & 0.9887315 & 2.9319240 \\
\hline $85 \mathrm{H} \mathrm{H} 48$ & 2.2310503 & 3.4327438 & -0.3603208 \\
\hline $86 \mathrm{H}$ H49 & 3.4117858 & 2.4835209 & -1.2165070 \\
\hline
\end{tabular}


$\underline{1+\mathrm{DABCO}}$

\begin{tabular}{|c|c|c|c|}
\hline \multirow[b]{2}{*}{ Atom } & \multicolumn{3}{|c|}{ Cartesian Coordinates (Angstroms) } \\
\hline & $X$ & $\mathrm{Y} \quad \mathrm{Z}$ & \\
\hline $1 \mathrm{C}$ C16 & 1.2089310 & -1.5514649 & -0.1404124 \\
\hline $2 \mathrm{~S} \mathrm{~S} 1$ & 0.8434249 & -3.1945276 & -0.1898216 \\
\hline $3 \mathrm{~N} \mathrm{~N} 2$ & 2.4839571 & -1.0697456 & -0.1091368 \\
\hline $4 \mathrm{H} \mathrm{H} 10$ & 2.5995125 & -0.0695143 & -0.2185575 \\
\hline $5 \mathrm{~N} \mathrm{~N} 3$ & 0.2805404 & -0.5364245 & -0.1334519 \\
\hline $6 \mathrm{H} \mathrm{H} 27$ & 0.6405493 & 0.4273059 & -0.1680656 \\
\hline $7 \mathrm{C} \mathrm{C} 17$ & -1.1231022 & -0.6064949 & -0.1231761 \\
\hline $8 \mathrm{C} \mathrm{C} 18$ & -3.9363673 & -0.4603232 & -0.0478571 \\
\hline $9 \mathrm{C}$ C19 & -1.8078393 & 0.4069801 & -0.8032659 \\
\hline $10 \mathrm{C} \mathrm{C} 20$ & -1.8601259 & -1.5672325 & 0.5860731 \\
\hline $11 \mathrm{C} \mathrm{C} 21$ & -3.2515290 & -1.4878668 & 0.6055873 \\
\hline $12 \mathrm{C} \mathrm{C} 22$ & -3.1983255 & 0.4888863 & -0.7479374 \\
\hline $13 \mathrm{H} \mathrm{H} 28$ & -1.2475707 & 1.1302262 & -1.3853439 \\
\hline $14 \mathrm{H} \mathrm{H} 30$ & -1.3468037 & -2.3653039 & 1.1036452 \\
\hline $15 \mathrm{H} \mathrm{H} 33$ & -5.0167641 & $1 \quad-0.4085715$ & $5-0.0159565$ \\
\hline $16 \mathrm{C} \mathrm{C} 23$ & 3.6965505 & -1.8707133 & -0.2670929 \\
\hline $17 \mathrm{C} \mathrm{C} 24$ & 5.4163840 & -2.7844409 & -1.8990332 \\
\hline $18 \mathrm{C} \mathrm{C} 25$ & 6.1208369 & -2.1025689 & 0.4379769 \\
\hline 19 C C26 & 6.5465478 & -2.2095660 & -1.0337670 \\
\hline $20 \mathrm{C} \mathrm{C} 27$ & 4.8250903 & -1.2914282 & 0.5990594 \\
\hline $21 \mathrm{C} \mathrm{C} 28$ & 4.1212951 & -1.9734941 & -1.7429378 \\
\hline $22 \mathrm{H} \mathrm{H} 36$ & 5.2260834 & -3.8256228 & -1.6051630 \\
\hline $23 \mathrm{H}$ H37 & 5.9612217 & -3.1111195 & 0.8431719 \\
\hline $24 \mathrm{H} \mathrm{H} 38$ & 6.8160151 & -1.2103320 & -1.4057236 \\
\hline $25 \mathrm{H} \mathrm{H} 39$ & 5.0145221 & -0.2480021 & 0.3004993 \\
\hline $26 \mathrm{H} \quad \mathrm{H} 40$ & 4.2744714 & -0.9583744 & -2.1393945 \\
\hline $27 \mathrm{H} \quad \mathrm{H} 41$ & 3.4393165 & -2.8710308 & 0.0937622 \\
\hline $28 \mathrm{H} \mathrm{H} 42$ & 5.7153918 & -2.8097876 & -2.9532068 \\
\hline $29 \mathrm{H} \mathrm{H} 43$ & 6.9195667 & -1.6499140 & 1.0368580 \\
\hline $30 \mathrm{H} \mathrm{H} 44$ & 7.4472147 & -2.8280928 & -1.1219844 \\
\hline $31 \mathrm{H} \mathrm{H} 45$ & 4.50 & -1.2703692 & 1.6483144 \\
\hline $32 \mathrm{H} \mathrm{H} 46$ & 3.3067623 & -2.4256999 & -2.3177468 \\
\hline 33 C C29 & -3.8628339 & 1.6414512 & -1.4475148 \\
\hline $34 \mathrm{C}$ C30 & -4.0248454 & -2.4831993 & 1.4323407 \\
\hline $35 \mathrm{~F} \mathrm{~F} 1$ & -5.3007159 & -2.6062559 & 1.0033055 \\
\hline $36 \mathrm{~F} \mathrm{~F} 2$ & -3.4637370 & -3.7081607 & 1.4066213 \\
\hline $37 \mathrm{~F} \mathrm{~F} 3$ & -4.0813457 & -2.1009652 & 2.7308812 \\
\hline 38 F F4 & -5.2067203 & 1.5672760 & -1.4105526 \\
\hline $39 \mathrm{~F} \mathrm{~F} 5$ & -3.508 & 2.8255572 & -0.8790857 \\
\hline $40 \mathrm{~F} \mathrm{F6}$ & -3.4904649 & 1.7191521 & -2.7451072 \\
\hline 41 N N1 & 1.7151695 & 4.6739524 & 1.2927295 \\
\hline $42 \mathrm{C} \mathrm{C} 1$ & 1.3708245 & 3.7165088 & 2.3580458 \\
\hline $43 \mathrm{H} \mathrm{H} 1$ & 0.5246207 & 4.1222409 & 2.9221435 \\
\hline 44 H H4 & 2.2209661 & 3.6473364 & 3.0450267 \\
\hline $45 \mathrm{C} \mathrm{C} 2$ & 0.5816590 & 4.7728135 & 0.3582372 \\
\hline $46 \mathrm{H} \mathrm{H} 3$ & -0.2885464 & 5.1329969 & 0.9165792 \\
\hline $47 \mathrm{H} \mathrm{H7}$ & 0.8213070 & 5.5280997 & -0.3977503 \\
\hline $48 \mathrm{C} \mathrm{C} 3$ & 2.8906327 & 4.1757897 & 0.5619933 \\
\hline $49 \mathrm{H} \mathrm{H} 2$ & 3.1281182 & 4.8907931 & -0.2329674 \\
\hline $50 \mathrm{H}$ H9 & 3.7438368 & 4.1539792 & 1.2483343 \\
\hline
\end{tabular}




$\begin{array}{lccc}\text { 51 C C4 } & 1.0234908 & 2.3222680 & 1.7437244 \\ \text { 52 H H5 } & -0.0134969 & 2.0350674 & 1.9452258 \\ \text { 53 H H11 } & 1.6649400 & 1.5290914 & 2.1428162 \\ \text { 54 C C5 } & 2.5997029 & 2.7557482 & -0.0261382 \\ \text { 55 H H12 } & 3.2678858 & 2.0048223 & 0.4133317 \\ \text { 56 H H13 } & 2.7378696 & 2.7304746 & -1.1127498 \\ \text { 57 C C6 } & 0.2926558 & 3.3842379 & -0.2965406 \\ \text { 58 H H6 } & 0.4519217 & 3.4090504 & -1.3802941 \\ \text { 59 H H14 } & -0.7396619 & 3.0674558 & -0.1226144 \\ \text { 60 N N5 } & 1.2031124 & 2.3573292 & 0.2707544\end{array}$

\section{1 + PMDETA}

\begin{tabular}{|c|c|c|c|}
\hline \multirow[b]{2}{*}{ Atom } & \multicolumn{3}{|c|}{ Cartesian Coordinates (Angstroms) } \\
\hline & $X$ & $\mathrm{Z}$ & \\
\hline $1 \mathrm{C} \mathrm{C} 16$ & 0.9400661 & -1.6040524 & -0.1443843 \\
\hline $2 \mathrm{~S} \mathrm{~S} 1$ & 0.6157847 & -3.2162549 & 0.2401523 \\
\hline $3 \mathrm{~N} \mathrm{~N} 2$ & 2.2057303 & -1.1049881 & -0.2575092 \\
\hline 4 H H10 & 2.3141278 & -0.0911790 & -0.2229078 \\
\hline $5 \mathrm{~N} \mathrm{~N} 3$ & -0.0108788 & -0.6399799 & -0.3807524 \\
\hline $6 \mathrm{H} \mathrm{H} 27$ & 0.3343899 & 0.2770472 & -0.6868464 \\
\hline $7 \mathrm{C} \mathrm{C} 17$ & -1.4092661 & -0.6787211 & -0.2598552 \\
\hline $8 \mathrm{C} \mathrm{C} 18$ & -4.2218703 & -0.4910748 & -0.1243812 \\
\hline 9C C19 & -2.0383473 & 0.5523458 & -0.0232264 \\
\hline $10 \mathrm{C} \mathrm{C} 20$ & -2.2035871 & -1.8246631 & -0.4249482 \\
\hline $11 \mathrm{C} \mathrm{C} 21$ & -3.5913786 & -1.7169660 & -0.3403202 \\
\hline $12 \mathrm{C} \mathrm{C} 22$ & -3.4281184 & 0.6437516 & 0.0248336 \\
\hline $13 \mathrm{H} \mathrm{H} 28$ & -1.4219558 & 1.4304084 & 0.1326002 \\
\hline $14 \mathrm{H} \mathrm{H} 30$ & -1.7344506 & $5-2.7785990$ & -0.6159406 \\
\hline $15 \mathrm{H} \mathrm{H} 33$ & -5.3010874 & H -0.4212929 & -0.0872832 \\
\hline $16 \mathrm{C} \mathrm{C} 23$ & 3.4183721 & -1.9007813 & -0.0703383 \\
\hline $17 \mathrm{C} \mathrm{C} 24$ & 5.7 & -2 & 603 \\
\hline $18 \mathrm{C} \mathrm{C} 25$ & 5.2956182 & -2.5397073 & 0724 \\
\hline $19 \mathrm{C} \mathrm{C} 26$ & 6.3278211 & -2.2347944 & 0.4230170 \\
\hline $20 \mathrm{C} \mathrm{C} 27$ & 4.0166113 & -1.7098841 & 9931 \\
\hline $21 \mathrm{C} \mathrm{C} 28$ & 4.4496489 & -1.6002796 & -1.1700992 \\
\hline $22 \mathrm{H} \mathrm{H} 36$ & 5.4894708 & -3.4951540 & -1.1209284 \\
\hline $23 \mathrm{H} \mathrm{H} 37$ & 5.0368427 & -3.6067652 & 1.4842987 \\
\hline $24 \mathrm{H} \mathrm{H} 38$ & 6.6 & 1208 & 0.5269876 \\
\hline $25 \mathrm{H} \mathrm{H} 39$ & 4.24 & -0.6 & 057 \\
\hline $26 \mathrm{H} \mathrm{H} 40$ & 4.7079942 & -0.5325104 & -1.1504086 \\
\hline $27 \mathrm{H} \mathrm{H} 41$ & 3.1001953 & -2.9429187 & -0.1617691 \\
\hline $28 \mathrm{H} \mathrm{H} 42$ & 6.4607054 & -2.1711481 & -1.7482931 \\
\hline $29 \mathrm{H} \mathrm{H} 43$ & 5.7220720 & -2.3553794 & 2.5108448 \\
\hline $30 \mathrm{H} \mathrm{H} 44$ & 7.2138715 & -2.8683412 & 0.5475669 \\
\hline $31 \mathrm{H} \mathrm{H} 45$ & 3.2636358 & -1.9894235 & 2.0795107 \\
\hline $32 \mathrm{H} \mathrm{H} 46$ & 4.0035875 & -1.8023337 & -2.1506696 \\
\hline 33 C C29 & -4.0458593 & 1.9966866 & 854 \\
\hline 34 C C30 & -4.4263740 & -2.9663093 & -0.4550219 \\
\hline $35 \mathrm{~F} \mathrm{~F} 1$ & -5.6740186 & -2.6933512 & -0.9032544 \\
\hline $36 \mathrm{~F} \mathrm{~F} 2$ & -3.8798602 & -3.8647042 & -1.3008540 \\
\hline $37 \mathrm{~F} \mathrm{~F} 3$ & -4.5643934 & -3.5810240 & 0.7412260 \\
\hline $38 \mathrm{~F} \mathrm{~F} 4$ & -5.3903503 & 1.9704658 & 0.1667490 \\
\hline 39 F F5 & -3.7241858 & 2.5068657 & 1.4596089 \\
\hline 40 F F6 & -3.6055217 & 2.8959611 & -0.6722761 \\
\hline $41 \mathrm{~N} \mathrm{~N} 1$ & 2.8609581 & 2.3232232 & 0.1154391 \\
\hline
\end{tabular}




$\begin{array}{lccc}\text { 42 C C1 } & 2.8324698 & 2.8795208 & 1.4825166 \\ \text { 43 H H4 } & 3.3972421 & 3.8318341 & 1.5240012 \\ \text { 44 H H5 } & 3.3833560 & 2.1789626 & 2.1187197 \\ \text { 45 C C13 } & 2.1499982 & 3.1758319 & -0.8518909 \\ \text { 46 C C3 } & 4.2707428 & 2.1880077 & -0.2664492 \\ \text { 47 H H3 } & 4.7820072 & 1.5175267 & 0.4301727 \\ \text { 48 H H11 } & 4.3692558 & 1.7649898 & -1.2679621 \\ \text { 49 C C4 } & 1.8813542 & 2.4867485 & -2.1994069 \\ \text { 50 H H12 } & 2.6878678 & 1.7830710 & -2.4216918 \\ \text { 51 H H13 } & 1.9043376 & 3.2447585 & -3.0040595 \\ \text { 52 C C5 } & 1.4701625 & 3.1761508 & 2.1151257 \\ \text { 53 H H14 } & 0.9893280 & 4.0149546 & 1.5984064 \\ \text { 54 H H15 } & 1.6979940 & 3.5487139 & 3.1362736 \\ \text { 55 N N4 } & 0.6135739 & 1.7393036 & -2.2390926 \\ \text { 56 N N5 } & 0.5113127 & 2.0753433 & 2.1537552 \\ \text { 57 C C7 } & 0.6267775 & 0.7602926 & -3.3320572 \\ \text { 58 H H17 } & -0.3204841 & 0.2153006 & -3.3448300 \\ \text { 59 H H21 } & 1.4317413 & 0.0383152 & -3.1681518 \\ \text { 60 H H22 } & 0.7680299 & 1.2284546 & -4.3219965 \\ \text { 61 C C8 } & -0.5242007 & 2.6520569 & -2.4038536 \\ \text { 62 H H18 } & -0.4622074 & 3.2210297 & -3.3488124 \\ \text { 63 H H23 } & -0.5689471 & 3.3689083 & -1.5800036 \\ \text { 64 H H24 } & -1.4605974 & 2.0905281 & -2.3978799 \\ \text { 65 C C11 } & 1.0194452 & 0.9197221 & 2.8888881 \\ \text { 66 H H2 } & 0.2541795 & 0.1394243 & 2.9140273 \\ \text { 67 H H32 } & 1.2893103 & 1.1646713 & 3.9346331 \\ \text { 68 H H34 } & 1.8998185 & 0.5035958 & 2.3953640 \\ \text { 69 C C12 } & -0.7404767 & 2.5366942 & 2.7525669 \\ \text { 70 H H1 } & -1.1437064 & 3.3791766 & 2.1825798 \\ \text { 71 H H19 } & -0.6162055 & 2.8633433 & 3.8027732 \\ \text { 72 H H47 } & -1.4825717 & 1.7356678 & 2.7315969 \\ \text { 73 H H48 } & 1.1920571 & 3.4624643 & -0.4169104 \\ \text { 74 H H49 } & 2.7141533 & 4.1115312 & -1.0283118 \\ \text { 75 H H7 } & 4.8012259 & 3.1582719 & -0.2578458\end{array}$

\section{$\underline{1+\text { TMEDA }}$}

\begin{tabular}{cccc}
\multicolumn{5}{c}{ Cartesian Coordinates (Angstroms) } \\
Atom & X & Y & Z \\
----- & ----------- & \\
1 C C16 & 1.1570678 & 1.2675919 & 0.0222826 \\
2 S S1 & 0.9494085 & 2.9278905 & -0.1958706 \\
3 N N2 & 2.3798598 & 0.6712669 & 0.0681768 \\
4 H H10 & 2.4168497 & -0.3418869 & -0.0773393 \\
5 N N3 & 0.1397676 & 0.3487417 & 0.1844900 \\
6 H H27 & 0.4285296 & -0.5907202 & 0.4873435 \\
7 C C17 & -1.2463911 & 0.4684560 & 0.0007023 \\
8 C C18 & -4.0591293 & 0.4444004 & -0.3149723 \\
9 C C19 & -1.9394608 & -0.7225780 & -0.2674805 \\
10 C C20 & -1.9850429 & 1.6566730 & 0.1120983 \\
11 C C21 & -3.3702227 & 1.6287463 & -0.0599405 \\
12 C C22 & -3.3234370 & -0.7358615 & -0.4065835 \\
13 H H28 & -1.3856709 & -1.6495293 & -0.3627610 \\
14 H H30 & -1.4722421 & 2.5833396 & 0.3279167 \\
15 H H33 & -5.1343914 & 0.4411501 & -0.4313642 \\
16 C C23 & 3.6490887 & 1.3944767 & -0.0070741 \\
17 C C24 & 6.0307823 & 1.4967964 & 0.8554797
\end{tabular}




\begin{tabular}{|c|c|c|c|}
\hline $18 \mathrm{C} \mathrm{C} 25$ & 5.5076252 & 2.2557995 & -1.5057231 \\
\hline $19 \mathrm{C} \mathrm{C} 26$ & 6.5519716 & 1.6083247 & -0.5845087 \\
\hline $20 \mathrm{C} \mathrm{C} 27$ & 4.1672180 & 1.5070433 & -1.4515880 \\
\hline $21 \mathrm{C} \mathrm{C} 28$ & 4.6921368 & 0.7433315 & 0.9151919 \\
\hline $22 \mathrm{H} \mathrm{H} 36$ & 5.8905068 & 2.5048970 & 1.2687728 \\
\hline $23 \mathrm{H} \mathrm{H} 37$ & 5.3486534 & 3.2986839 & -1.1992757 \\
\hline $24 \mathrm{H} \mathrm{H} 38$ & 6.7925174 & 0.6028516 & -0.9597954 \\
\hline 25 H H39 & 4.2967586 & 0.4965878 & -1.8645439 \\
\hline $26 \mathrm{H} \mathrm{H} 40$ & 4.8532592 & -0.3016587 & 0.6106266 \\
\hline 27 H H41 & 3.4402613 & 2.4057996 & 0.3544496 \\
\hline $28 \mathrm{H} \mathrm{H} 42$ & 6.7684484 & 0.9975785 & 1.4947389 \\
\hline $29 \mathrm{H} \mathrm{H} 43$ & 5.8759867 & 2.2893798 & -2.5376754 \\
\hline $30 \mathrm{H} \mathrm{H} 44$ & 7.4864250 & 2.1809629 & -0.6053528 \\
\hline $31 \mathrm{H} \mathrm{H} 45$ & 3.4120860 & 2.0149830 & -2.0592718 \\
\hline $32 \mathrm{H} \mathrm{H} 46$ & 4.3094525 & 0.7201953 & 1.9423092 \\
\hline 33 C C29 & -3.9890580 & -2.0548644 & -0.6841672 \\
\hline 34 C C30 & -4.1151725 & 2.9351552 & 0.0475203 \\
\hline 35 F F1 & -5.4541487 & 2.7654965 & -0.0240372 \\
\hline $36 \mathrm{~F} F 2$ & -3.8500760 & 3.5608646 & 1.2160605 \\
\hline $37 \mathrm{~F}$ F3 & -3.7646131 & 3.7875439 & -0.9398024 \\
\hline $38 \mathrm{~F}$ F4 & -5.3327373 & -1.9729156 & -0.6611308 \\
\hline $39 \mathrm{~F} \mathrm{~F} 5$ & -3.6323375 & -2.5501557 & -1.8920969 \\
\hline $40 \mathrm{~F} \mathrm{~F} 6$ & -3.6250475 & -2.9925675 & 0.2316921 \\
\hline $41 \mathrm{~N}$ N1 & 2.4744326 & -2.4736675 & -0.7590856 \\
\hline $42 \mathrm{C} \mathrm{C} 1$ & 1.4257689 & -2.6453258 & -1.7682042 \\
\hline $43 \mathrm{H} \mathrm{H} 4$ & 1.4777839 & -3.6292553 & -2.2680308 \\
\hline $44 \mathrm{H} \mathrm{H}$ H & 1.5248856 & -1.8680463 & -2.5305927 \\
\hline $45 \mathrm{C} \mathrm{C} 13$ & 2.4072905 & -3.4928987 & 0.2970583 \\
\hline $46 \mathrm{C} \mathrm{C} 3$ & 3.7886831 & -2.5355159 & -1.4060932 \\
\hline $47 \mathrm{H} \mathrm{H} 3$ & 3.8584624 & -1.7634978 & -2.1766666 \\
\hline $48 \mathrm{H} \mathrm{H} 11$ & 4.5778705 & -2.3578008 & -0.6696665 \\
\hline $49 \mathrm{C} \mathrm{C} 4$ & 1.1151918 & -3.5298157 & 1.1183454 \\
\hline $50 \mathrm{H} \mathrm{H} 12$ & 1.2566766 & -4.3107199 & 1.8900617 \\
\hline $51 \mathrm{H} \mathrm{H} 13$ & 0.2841926 & -3.8723424 & 0.4923204 \\
\hline $52 \mathrm{~N} \mathrm{~N} 4$ & 0.7107028 & -2.2572025 & 1.7318656 \\
\hline $53 \mathrm{C} \mathrm{C7}$ & 1.7242025 & -1.7384717 & 2.6566403 \\
\hline $54 \mathrm{H} \mathrm{H} 17$ & 1.3706376 & -0.7971667 & 3.0857834 \\
\hline $55 \mathrm{H} \mathrm{H} 21$ & 2.6553437 & -1.5311150 & 2.1275283 \\
\hline $56 \mathrm{H} \mathrm{H} 22$ & 1.9358424 & -2.4377437 & 3.4850554 \\
\hline $57 \mathrm{C} \mathrm{C} 8$ & -0.5607377 & -2.4327048 & 2.4463774 \\
\hline $58 \mathrm{H} \mathrm{H} 18$ & -0.4708125 & -3.1394883 & 3.2900595 \\
\hline $59 \mathrm{H} \mathrm{H} 23$ & -1.3335598 & -2.8056676 & 1.7693349 \\
\hline $60 \mathrm{H} \mathrm{H} 24$ & -0.8937992 & -1.4696026 & 2.8423802 \\
\hline $61 \mathrm{H} \mathrm{H} 48$ & 2.5410912 & -4.5074014 & -0.1261331 \\
\hline 62 H H49 & 3.2616450 & -3.3258903 & 0.9616365 \\
\hline $63 \mathrm{H} \mathrm{H} 7$ & 3.9791157 & -3.5137009 & -1.8831872 \\
\hline 64 H H51 & 0.4370790 & -2.5424611 & -1.3193358 \\
\hline
\end{tabular}

\section{SI References}

(1) Webb, J. E. A.; Crossley, M. J.; Turner, P.; Thordarson, P. J. Am. Chem. Soc. 2007, 129, 7155 .

(2) Thordarson, P. Chem. Soc. Rev. 2011, 40, 1305.

(3) Deranleau, D. A. J. Am. Chem. Soc. 1969, 91, 4044. 
(4) Horman, I.; Dreux, B. Anal. Chem. 1983, 55, 1219.

(5) Peters, S. J.; Stevenson, C. D. J. Chem. Educ. 2004, 81, 715. 